\title{
Numerical investigation on the performance of a small counter-rotating wind turbine
}

\author{
Michat Pacholczyk ${ }^{1, *}$, Krzysztof Blecharz ${ }^{1}$, and Dariusz Karkosiński ${ }^{1}$ \\ ${ }^{1}$ Gdańsk University of Technology, Faculty of Electrical and Control Engineering, Gdańsk, Poland
}

\begin{abstract}
The article presents results of the investigation on the performance of a small counter-rotating wind turbine. Wind turbine has been simulated using Computational Fluid Dynamics methods. Actuator Line Model has been successfully used to represent rotors in computational domain. Parametric study has been carried out, taking into account changes in the tip speed ratio of the rotors while maintaining a constant distance between upwind and downwind rotor. Study results revealed noticeable increase in power coefficient for optimal configuration. Dynamic interaction between rotors has been investigated exposing no significant interference in both torque and power.
\end{abstract}

\section{Introduction}

Wind turbines have become one of the most popular renewable energy sources and have been extensively developed in recent decades [1]. Definitely the most often, single rotor 3-bladed Horizontal Axis Wind Turbines (HAWT) are studied or in an operation. However, those are not the only types ever designed. Due to its capability to operate independently on a wind direction Vertical Axis Wind Turbines have gained some industry's interest. Instead of airfoil lift force, Magnus effect can be utilized to produce torque on a turbine's shaft. Thanks to possibility to increase a power coefficient Counter-Rotating Wind Turbine (CRWT) concept is also being widely investigated.

Single rotor HAWT has a theoretical wind energy extraction efficiency limited to $59.3 \%$ as Betz's classical theory stated [2]. The limit is achieved when axial induction factor is equal to $1 / 3$, thus wind velocity is reduced to $2 / 3$ of a value upwind. CRWT concept assumes that part of the energy left in a wake can be extracted by the second rotor. Theoretical basis have been delivered by Newman in [3] and extended in [4]. Author stated that maximal power coefficient $\mathrm{C}_{\mathrm{p}}$ for 2 rotor discs is $64 \%$ and for infinite number $\sim 66.7 \%$. When considered that modern HAWTs operate at the efficiency level of 40-50\% [5] CRWTs can provide affordable supplementation for collection of renewable energy sources.

CRWT are being investigated experimentally and numerically. The former includes wind tunnel testing (see e.g. [6, 7]) as well as field test, for example [8] or study of $6 \mathrm{~kW}$ CRWT carried out over a period of 4 month [9]. The later are mainly performed with use of Computational Fluids Dynamics (CFD). However, some simpler methods like those presented in [10] and [11] have been proposed and used. Different approaches are considered

* Corresponding author: michal.pacholczyk@pg.edu.pl 
when simulating wind turbines with CFD. General differentiation between most common methods has been described in [12]. In the present study one of the simplified actuators methods is used since direct rotor representation is computationally expensive (nevertheless some authors used this approach for CRWT simulation, see for example [13]). Numbers of studies with one of such a method have been performed investigating SRWT operation. Obtained results verified its viability (see for example [14-16] or [17]). Also, few trials with CRWT have been previously conducted, even including CRWT wind farm simulation [18]. Shen et al. studied in [19] different turbine's configurations and used results to predicate annual energy gain. But in all configurations they have assumed that up and downwind rotor are accurately counter-rotating. More extensive parametric study has been presented in [20]. However, in this investigation authors utilised Actuator Disc Model which does not take into account single blade influence and thus does not produce blade's tip vortices. Moreover, study was conducted in steady state using averaged turbulence model which neglects dynamic interactions.

More comprehensive study on current CRWT development stage is presented in [21] nevertheless from literature review it can be seen that noticeable increase in turbine's efficiency is being reported. Therefore, numerical parametric study of small CRWT have been carried out and its findings have been presented in this paper. But, in contrast to [19] combination of rotors' tip speed ratios was in main interest. Axial distance between rotors has been arbitrary set to $0.5 \mathrm{D}$. In order to include dynamic effects unsteady simulations were carried out using Large Eddy Scale method and appropriate sub-grid modelling.

\section{Methodology}

In this study Computational Fluid Dynamics methods have been used in order to investigate CRWT performance. One of the turbulent flows simulation method used in CFD is Large Eddy Scale (LES). In this approach only large turbulent structures are numerically solved while smaller are modelled using sub-grid scale models. This kind of Navier-Stokes equations filtering converts them to form:

$$
\begin{gathered}
\nabla \cdot \widetilde{\boldsymbol{u}}=0 \\
\frac{\partial \widetilde{\boldsymbol{u}}}{\partial t}+(\widetilde{\boldsymbol{u}} \cdot \nabla) \widetilde{\boldsymbol{u}}=-\frac{1}{\rho} \nabla \tilde{p}+\nu \nabla^{2} \widetilde{\boldsymbol{u}}-\nabla \cdot \tau_{S G S}+\frac{1}{\rho} \overrightarrow{\boldsymbol{G}}
\end{gathered}
$$

where $\tau_{S G S}=-v_{S G S}\left(\nabla \widetilde{\boldsymbol{u}}+(\nabla \widetilde{\boldsymbol{u}})^{T}\right)$ and $v_{S G S}$ is the sub-grid scale viscosity. In present study it is modelled with standard Smagorinsky model [22]. $\widetilde{\boldsymbol{u}}$ is filtered velocity vector, $\tilde{p}$ is filtered pressure and $v$ is kinematic viscosity. $\overrightarrow{\boldsymbol{G}}$ is external forces vector.

In order to investigate wind turbine performance and wake one has to put its representation into computational domain. Wind turbine blades geometry can be included in grid in full form. However, this solution is computationally expensive since mesh close to the blade's surface has to be extremely dense (in order to properly simulate flow in boundary layer). A simpler alternatives are wind turbine models introduced into domain. Such a models apply a thrust and torque generated by blades on the flow but without resolving its full geometry. Several models have been developed including Actuator Disk Model [23] or Actuator Surface Model [24]. In this study Actuator Line Model (ALM) [25] has been used due to its capability to represent separated blades and thus to investigate dynamics interaction between rotors' blades. ALM's computational cost is also relatively low.

The ALM represents the blades as an actuator lines along each blade's axis. Those lines are discretized into set of discrete elements. Blade properties such as airfoil type, twist and 
chord length are determined in centre of each section. Airfoils look-up tables with lift and drag coefficients as a function of angle of attack are included into solver. Combined with the found flow velocity vector angle of attack, velocity magnitude and thus, lift and drag can be calculated for each actuator element. Lift and drag are then used to impose forces equal and opposite to them into a momentum equation. Force projection on surrounding finite elements is performed in form of the Gaussian function:

$$
\overrightarrow{\boldsymbol{f}}_{\text {proj }}=\frac{\overrightarrow{\boldsymbol{F}}}{\varepsilon^{3} \pi^{3 / 2}} \exp \left[-(r / \varepsilon)^{2}\right]
$$

where $\overrightarrow{\boldsymbol{F}}$ is calculated force, $\varepsilon$ Gaussian projection width and $r$ distance from actuator point.

Proper selection of projection width $\varepsilon$ is crucial for power production prediction. If wrong selected power can under or overestimated. Other important factors include number of the actuator points along blade's span, simulation time step and grid resolution. Sufficient number of actuation points ensures continuous distribution of force while short time step is required to maintain a smooth application of body force. Best practices and guidelines for simulation set up have been provided for example in [26].

\section{Simulation setup}

\subsection{Test wind turbine blade design}

In the present study small CRWT is under test. Both rotors are the same 3-bladed rotors and have diameter of $\mathrm{D}=1.4 \mathrm{~m}$. Root diameter is $0.25 \mathrm{~m}$. Rotor's blade was designed with help of Blade Element Momentum Theory (BEMT). Selected airfoil is NACA4418. Blade has been optimised for TSR $=5$. In the Figure 1 airfoil twist and chord distributions along blade's span have been presented. Right hand side of a figure presents selected airfoil. Detailed blade design process has been described in [27].
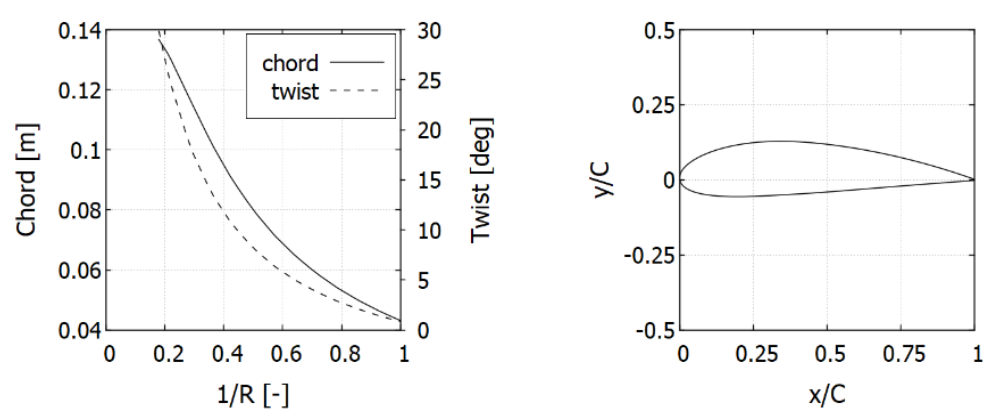

Fig. 1. Airfoils distribution along blade's span (left) and used NACA4418 profile (right).

\subsection{Computational setup}

Computational domain is presented in Figure 2. It consists of several regions with different grid refinements. Grid resolution gradually increases toward turbine placement. Refinement is used in order to provide sufficiently dense mesh grid in direct turbine proximity and to maintain relatively low total grid elements count (9.573 mln elements in total). Highest grid resolution region where CRWT is inserted has a total length of 4D. Domain has been 
discretized in space and with second order schemes. Boundary conditions have been set as presented in Figure 2. Large Eddy Scale method with standard Smagorinsky model has been used to solve unsteady turbulent flow equations. Equations are solved sequentially with PISO algorithm. SOFWA's implementation of ALM in OpenFOAM package has been used. Calculations were carried out at the Academic Computer Centre in Gdańsk.

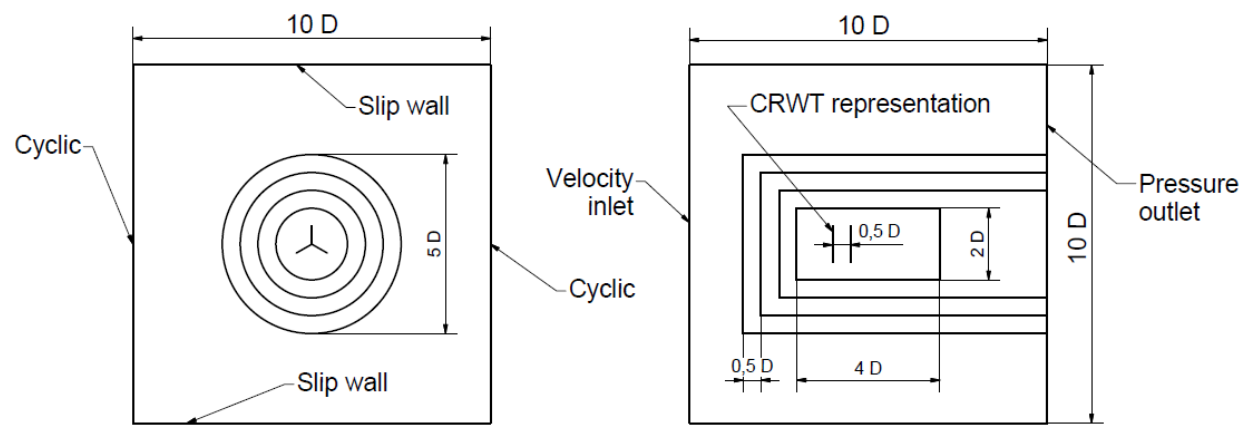

Fig. 2. Computational domain with boundary condition and grid refinement regions.

ALM model's parameters have been set as follows: Gaussian projection width $\varepsilon$ was set to $0.035 \mathrm{~m} ; 80$ actuator points were introduced along blade span; total simulation time was $5 \mathrm{~s}$ with time step equal to $0.0002 \mathrm{~s}$. It results in 25000 time steps for each CRWT configuration. During simulation with TSR $=5$ each rotor perform over 45 revolution.

\section{Results}

\subsection{ALM setup verification}

In order to verify ALM model set up, prior to the CRWT, Single Rotor Wind Turbine simulation has been performed. Results have been then confronted with BEMT calculations. This simple iterative method used to turbine performance predication is widely spread and accepted in wind energy field as it gives credible results (see for example [1, 28, 29]). Classical BEMT however, isn't appropriate for analysing CRWT (its modifications has been proposed in [10]). In the Figure 3 power, torque and thrust characteristics for both BEMT and ALM results have been compared. One can find them in a very good agreement for studied TSR range.
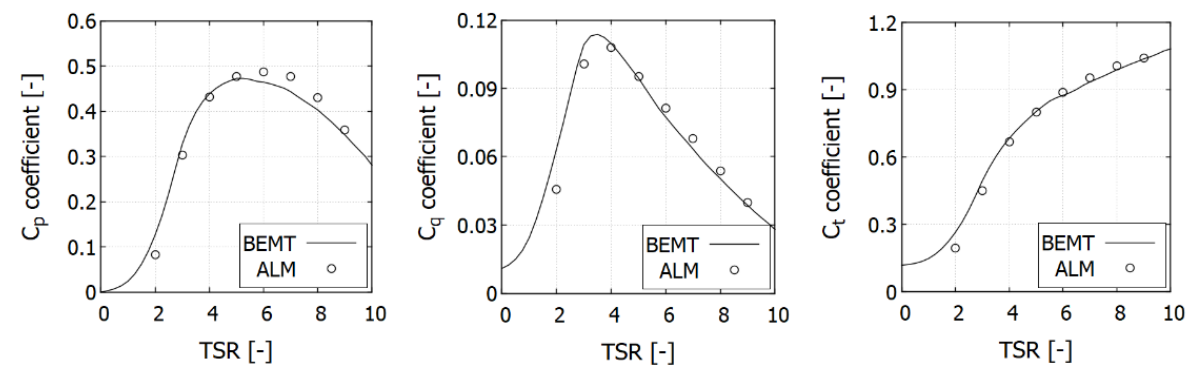

Fig. 3. BEMT and ALM calculation results comparison. 
Nevertheless it can be seen, that even tough blade was designed and optimised for TSR $=5$ operational optimum with ALM method is found around TSR $=6$ and beyond TSR $=5$ values are slightly overestimated. Differences are yet small enough to be accepted. With numerical setup validated, second rotor's representation has been added into a domain and CRTW parametrical simulation has been carried out.

\subsection{Small CRWT performance}

Upwind and downwind rotors were placed 0.5D from each other. Uniform input with wind velocity of $8 \mathrm{~m} / \mathrm{s}$ has been set up. Rotors' TSR varied from 2 to 8 with step equal to 1 (for both rotors TSR is calculated in relation to input wind speed). In total, 49 simulations were carried out. Results of this parametric study have been collected in form of maps presented in Figure 4. Sums of rotors power $\left(C_{p}\right)$, torque $\left(C_{m}\right)$ and thrust $\left(C_{t}\right)$ coefficients occupy left hand side of the graph while their differences are presented at the right hand side (front rotor coefficient value minus rear one).
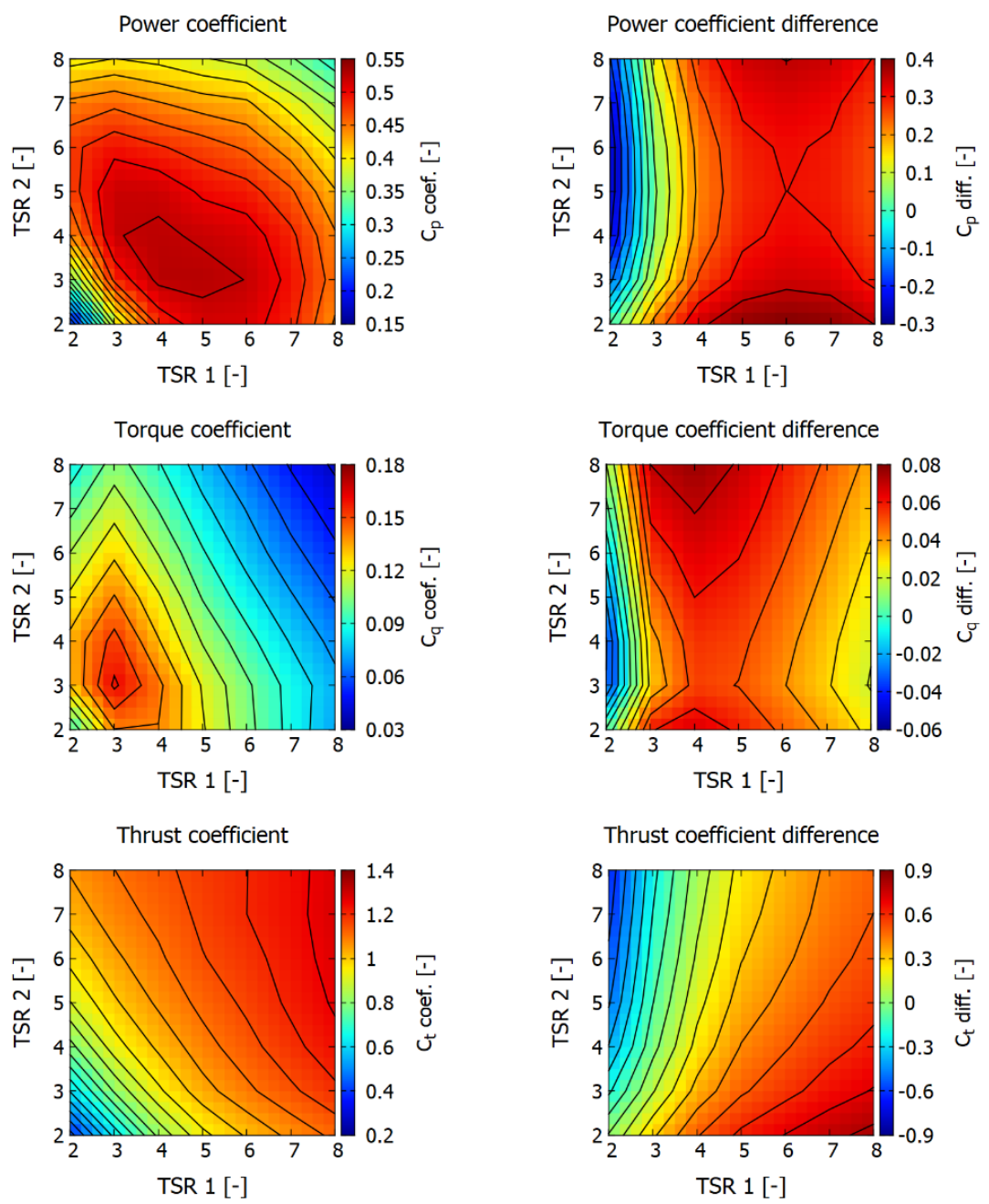

Fig. 4. Sum of front and rear rotor coefficient (left column) and their differences (right column). 
Peak CRWT power coefficient $\mathrm{C}_{\mathrm{p}}$ has been found for $\mathrm{TSR}_{1}=5$ and $\mathrm{TSR}_{2}=3$. This means that front rotor operates at the its characteristic optimum while second under that value. This finding shows that biggest increase in total CRWT coefficient $C_{p}$ shouldn't necessarily been expected when rotors are counter-rotating with the same speed. Total CRWT $\mathrm{C}_{\mathrm{p}}$ equals 0.5257 which is $5.95 \%$ more than in case of direct counter-rotation with TSR $=5$ and $10.29 \%$ more than single rotor wind turbine operating at the same conditions. Moreover, in comparison to SRWT, relative shaft rotational speed increased over $60 \%$. As expected and seen from $\mathrm{C}_{\mathrm{p}}$ difference map, front rotor produce most of the power in all studied TSR configuration. In optimum case front rotor $C_{p}$ is 0.4218 and rear is 0.104 . In comparison to SRWT, second rotor of CRWT reduced first rotor power of $11.52 \%$.

Taking into consideration torque and thrust one can see that optimums don't correspond to total power coefficient peak. As expected, thrust is lowest when both rotors barely rotate. In optimum case $C_{t}$ is equal to 1.0295 and is $28.71 \%$ higher than in SRWT. Torques coefficient is highest and equal to 0.1618 when both rotors directly counter-rotate but with TSR $=3$ and is higher over $49.91 \%$ in comparison 0.1079 in SRWT optimum. Lowest difference in rotors torque is obtained when TSR of front and rear rotor is 2 . In optimum operational point $\mathrm{C}_{\mathrm{q}}$ equals to 0.119 (24.82\% higher than SRWT).

Transient simulations were carried out therefore it was possible to analyse dynamic interaction between both rotors. In the Figure 5 CRWT power and torque plots from last $1 \mathrm{~s}$ of the optimum case simulation are presented. It can be clearly seen that no significant interference between front and rear rotor is observed for power nor torque and they can be considered as negligible. However one can see that interference exists and is excepted to increase with rotors close-up.

Mean and instantaneous wind speed contours and vorticity contour for optimum CRWT operation are shown in Figure 6. Again, not significant interference between rotors is visible but relatively high reduction in wind speed behind second rotor can be observed. It is noticeable that wake loose its stability just around 1D behind rear rotor. However, more detailed study is required in order to accurately investigate CRWT wake which was not a case in a present study (denser mesh should be established in wake region).
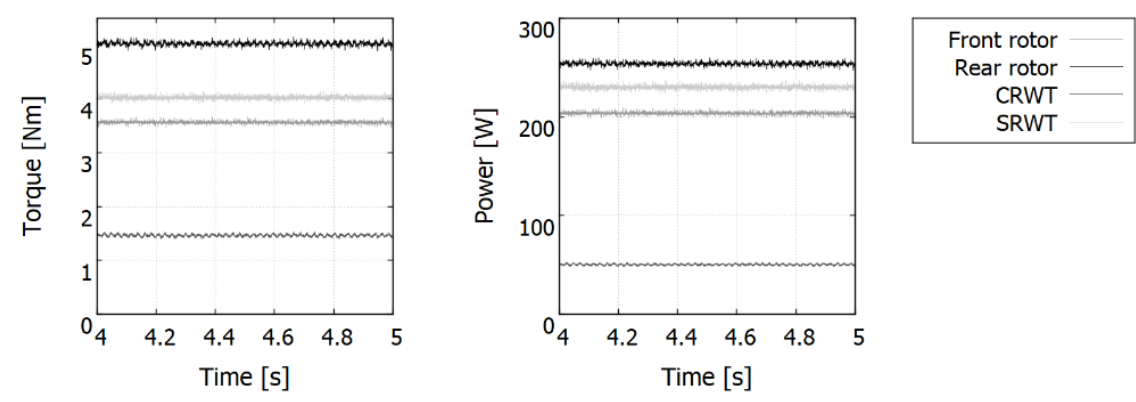

Fig. 5. Plots of a torque (left) and power (right) on the turbine shaft.

\section{Conclusion}

In this paper parametric CFD study on a small counter-rotating wind turbine performance has been carried out. Investigated parameters, e.g. front and rear rotors TSR have been varied over wide range revealing best operational conditions. It has turn out that peak performance is not obtained when both rotor are exactly counter-rotating but when front and rear rotor operate with TSR equal to 5 and 3 , respectively. Power coefficient in this configuration 
increased over $10.29 \%$ in comparison to SRWT at TSR $=5$, while relative rotational speed increased over $60 \%$. Simultaneously, total thrust increased over $28.71 \%$ and torque over $24.82 \%$. Interaction between rotors has been investigated exposing no significant dynamic interference in both torque and power plots. However, in terms of mean values rear rotor decrease front rotor power of almost $12 \%$ in comparison to SRWT.
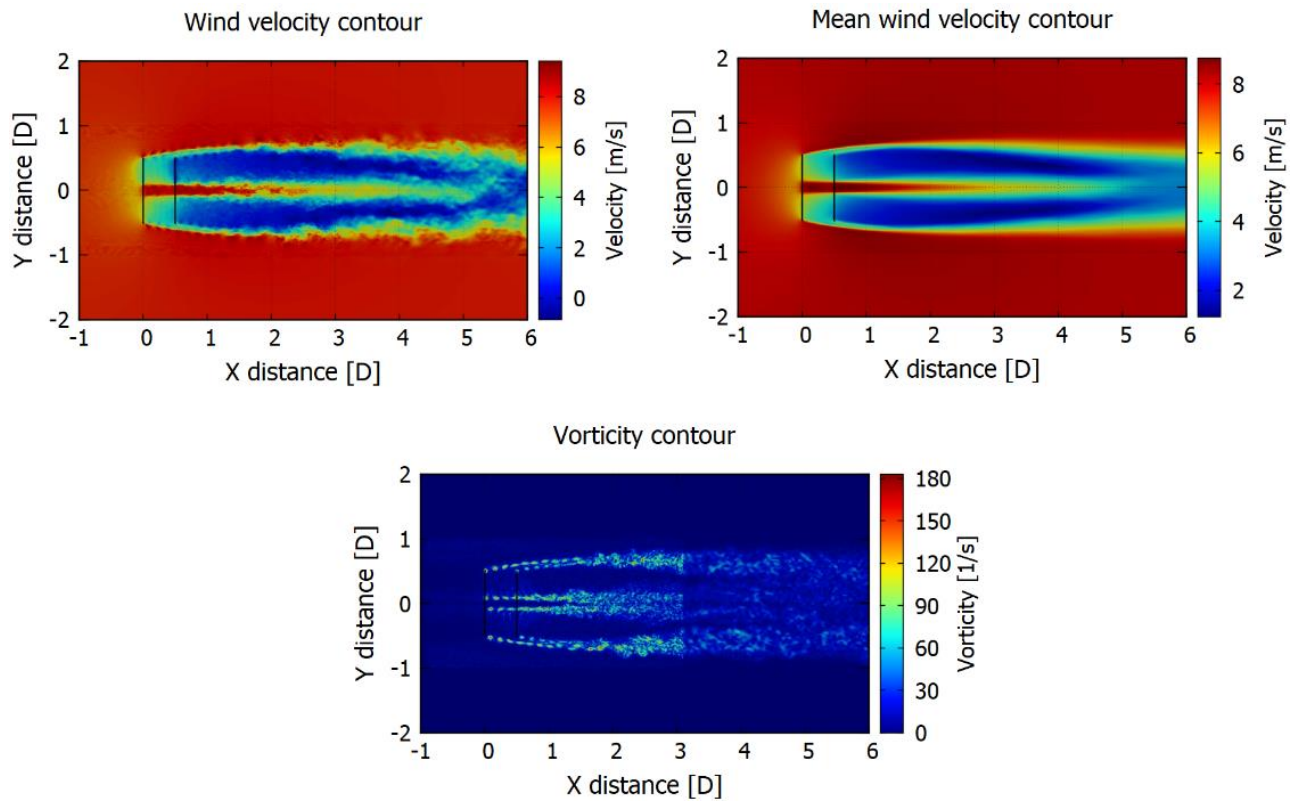

Fig. 6. Instantaneous (top left) and mean (top right) velocity and vorticity contour (bottom).

It has been shown that CRWT can be considered as affordable renewable energy source. Moreover, Actuator Line Model used in this study turn out to be computationally cost effective approach to CRWT simulations with ability to reveal dynamic interaction. Future study should include increase of investigated axial distances between rotors, accurate investigation of near and far CRWT wake as well as experimental verification of obtained results and ALM approach applied to CRWT.

\section{References}

1. X. Tang, X. Huang, R. Peng, X. Liu, Procedia CIRP 36, 12-16 (2015)

2. A. Betz, Introduction to the Theory of Flow Machines (1966)

3. B. G. Newman, J. Wind Eng. Ind. Aerodyn. 15, 1-3, 347-355 (1983)

4. B. G. Newman, J. Wind Eng. Ind. Aerodyn. 24, 3, 215-225 (1986)

5. P. J. Schubel, R. J. Crossley, Energies 5, 9, 3425-3449 (2012)

6. L. A. Mitulet, G. Oprina, R. A. Chihaia, S. Nicolaie, A. Nedelcu, M. Popescu, Procedia Eng. 100, 1141-1149 (2015)

7. S. Merchant, J. Gregg, K. Van Treuren, I. Gravagne, Proceedings of the 2009 ASEE Gulf-Southwest Annual Conference (2009) 
8. L. Romański, J. Bieniek, P. Komarnicki, M. Dębowski, J. Detyna, Eksploat. i Niezawodn. - Maint. Reliab. 18, 2, 201-209 (2016)

9. K. Appa, Counter Rotating Wind Turbine System. EISG Final Report (2002)

10. S. Lee, H. Kim, E. Son, S. Lee, Renew. Energy, 42, 140-144 (2012)

11. S. Lee, E. Son, S. Lee, Renew. Energy 54, 235-240 (2013)

12. B. Sanderse, S. P. Pijl, B. Koren, Wind Energy 14, 7, 799-819 (2011)

13. V. A. Koehuan, Sugiyono, S. Kamal, IOP Conf. Ser. Mater. Sci. Eng. 267, 1 (2017)

14. L. A. Martínez-Tossas, M. J. Churchfield, S. Leonardi, Wind Energy 18, 6, 1047-1060 (2015)

15. P.-E. Réthoré, P. van der Laan, N. Troldborg, F. Zahle, N. N. Sørensen, Wind Energy 17, 6, 919-937 (2014)

16. N. Troldborg, G. C. Larsen, H. A. Madsen, K. S. Hansen, J. N. Sørensen, R. Mikkelsen, Wind Energy 14, 7, 859-876 (2011)

17. J. N. Sørensen, R. F. Mikkelsen, S. Dan, S. Ivanell, S. Sarmast, S. J. Andersen, Phil. Trans. R. Soc. A 373, 2035 (2015)

18. A. Vasel-Be-Hagh, C. L. Archer, Sustain. Energy Technol. Assessments 24, 19-30 (2017)

19. W. Z. Shen, V. A. K. Zakkam, J. N. Sørensen, K. Appa, J. Phys. Conf. Ser. 75, 1 (2007)

20. A. Rosenberg, S. Selvaraj, A. Sharma, J. Phys. Conf. Ser. 524, 1 (2014)

21. G. Oprina, R. A. Chihaia, S. Nicolaie, C. A. Băbuțanu, A. Voina, J. Sustain. Energy 7, 3, 91-98 (2016)

22. J. Smagorinsky, Mon. Weather Rev. 91, 3, 99-164 (1963)

23. R. F. Mikkelsen, Actuator Disc Methods Applied to Wind Turbines (Technical University of Denmark, 2003)

24. W. Z. Shen, J. N. Sørensen, J. Zhang, Proceedings of European Wind Energy Conference 2007 (2007)

25. J. N. Sørensen W. Z. Shen, J. Fluids Eng. 124, 2, 393-399 (2002)

26. L. Martinez, S. Leonardi, M. Churchfield, P. Moriarty, 50 ${ }^{\text {th }}$ AIAA Aerosp. Sci. Meet. Incl. New Horizons Forum Aerosp. Expo. (2016)

27. M. Pacholczyk, K. Blecharz, Zesz. Nauk. Wydz. Elektrotechniki i Autom. Politech. Gdańskiej 61, 62-64 (2018)

28. C. Thumthae, Energy Procedia 75, 651-657 (2015)

29. F. Mahmuddin, Energy Procedia 105, 1123-1129 (2017) 